\title{
MODELLING AND SIMULATING A CONTROLLED PRESS-BRAKE SUPPLY SYSTEM
}

\author{
Lovrec, D. \& Kastrevc, M. \\ University of Maribor, Faculty of Mechanical Engineering, Smetanova 17, SI-2000 Maribor, Slovenia \\ E-Mail: d.lovrec@uni-mb.si
}

\begin{abstract}
Modern machinery and equipment manufacturers incorporate advanced technology into their products. This is especially true for those applications where an electro-hydraulic supply system is used, e.g. on tool and metal shaping/forming machines. Such machines represent a complex and multitechnological mechatronic system, and should be first modelled and simulated, especially in respect of dynamic behaviour.

This paper presents a theoretical analysis of an energy-saving and cost-effective electrohydraulic supply system on a hydraulic press-brake, used within the automotive industry. It emphasises the designing an adequate mathematical-simulation model, to serve as the basis for decisions concerning the used supply system's dynamic.

(Received in December 2010, accepted in June 2011. This paper was with the authors 2 months for 1 revision.)
\end{abstract}

Key Words: Metal Forming Machines, Electro Hydraulic System, Modelling, Simulation

\section{INTRODUCTION}

Reductions in energy consumption and noise reduction regarding drive systems, as well as cost-effectiveness, are increasingly important factors in modern machinery design. All of the above requirements are especially important for machines equipped with hydraulic supply and drive systems. Only the principle of volumetrically-controlled hydraulic energy (variable supply systems e.g. with the use of variable pumps), has proved satisfactory for the above requirements. In this regard, electro-hydraulic solutions in particular, make it possible to use all the advantages of modern electrical signal transmission and controller designs.

Such variable supply systems can be, in principle, controlled hydraulically or electrically. Constant-speed induction motors in combination with a variable displacement volume pump are commonly used solutions, in order to control flow, or consequently, the pressure of the medium. The second concept, for the same purpose, representing the application of a constant displacement volume pump in combination with variable rotational speed motors, has recently gained significance in praxis due to its attractive price. Such systems provide several advantages such as economical use of energy, user-friendly process control, and ease of maintenance, when compared to hydraulic-mechanical solutions.

In particular, electro hydraulic variable-speed constant pumps have recently been the object of some fundamental research work (see e.g. [1-3]), and work describing early applications of novel control principles (e.g. for use in Plastic Injection Moulding Machines [4-5]). The speed-controlled pump drive concept is still an attractive subject for detailed research [6-8].

In such solutions, the implementation of electric, electronic, electrical sensor and modern actuator technologies is very important for the evolution of intelligent electro-hydraulic systems. Such systems provide several advantages such as economical use of energy, userfriendly process control and ease of maintenance, compared to the hydraulic-mechanical solutions. However, from the designer's point of view they are more demanding. 
How to proof the dynamic behaviour of such a system using numerical simulation in order to check the feasibility of further experiments, or is it possible to replace an existing hydraulic supply system powered, for example by a hydraulic-mechanically controlled pump, with the speed-controlled constant pump, are common questions posed by designers of such as complex mechatronic systems.

The layout of a hydraulic press-brake with the used speed-controlled pump containing all the important system interactions of hydraulics, electronics, control techniques, and sensor technology, is shown in Fig. 1. It is advisable, when handling such a complex model to divide it into sub-systems: electric motor with frequency converter, constant pump, pipeline system with its own dynamic behaviour and an actuator, type of controller, and control strategy ... with all the most important characteristics and interactions, as shown in Fig. 1.
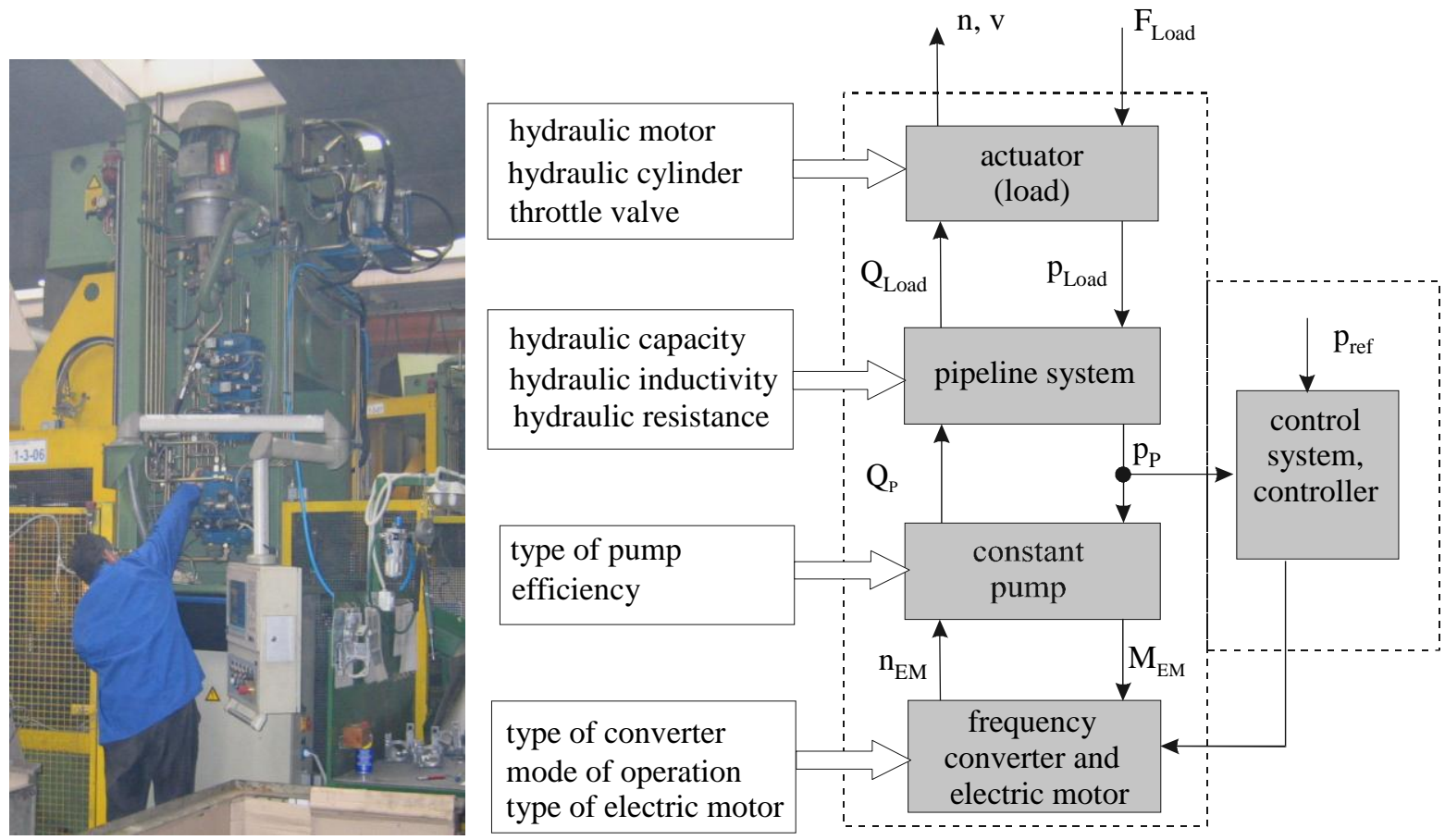

Figure 1: Layout of the press-brake and discussed supply system with interactions

The discussed system consists of a pump, driven by speed-controlled electric motor. The pump supplies fluid into the pipeline by which fluid is transported to the actuator. The pressure within the actuator corresponds to the type of actuator - load, whilst pressure within the pump depends on the static and dynamic behaviours of the pipeline. All the abovementioned entities must be considered whilst modelling a controlled-system.

\section{WORKING PROFILE OF HYDRAULIC PRESS BRAKE}

In order to carry-out the intended task, it was necessary to establish a real working-profile of the machine and its pressure characteristics, over the whole working cycle. The measured pressure-profiles describe the actual dynamics of the existing hydraulic supply system and actuator. A pump pressure-profile of the most dynamically-demanding when machining, was chosen - Fig. 2.

There are two pressure-values presented in Fig. 2, cylinder-pressure $\left(p_{c y l}\right)$ and pumppressure $\left(p_{1}\right)$ respectively. Cylinder-pressure provides information regarding the progress of the work and any corresponding forces on the cylinder. The pump pressure-profile is decisive for providing the required system's dynamics. 


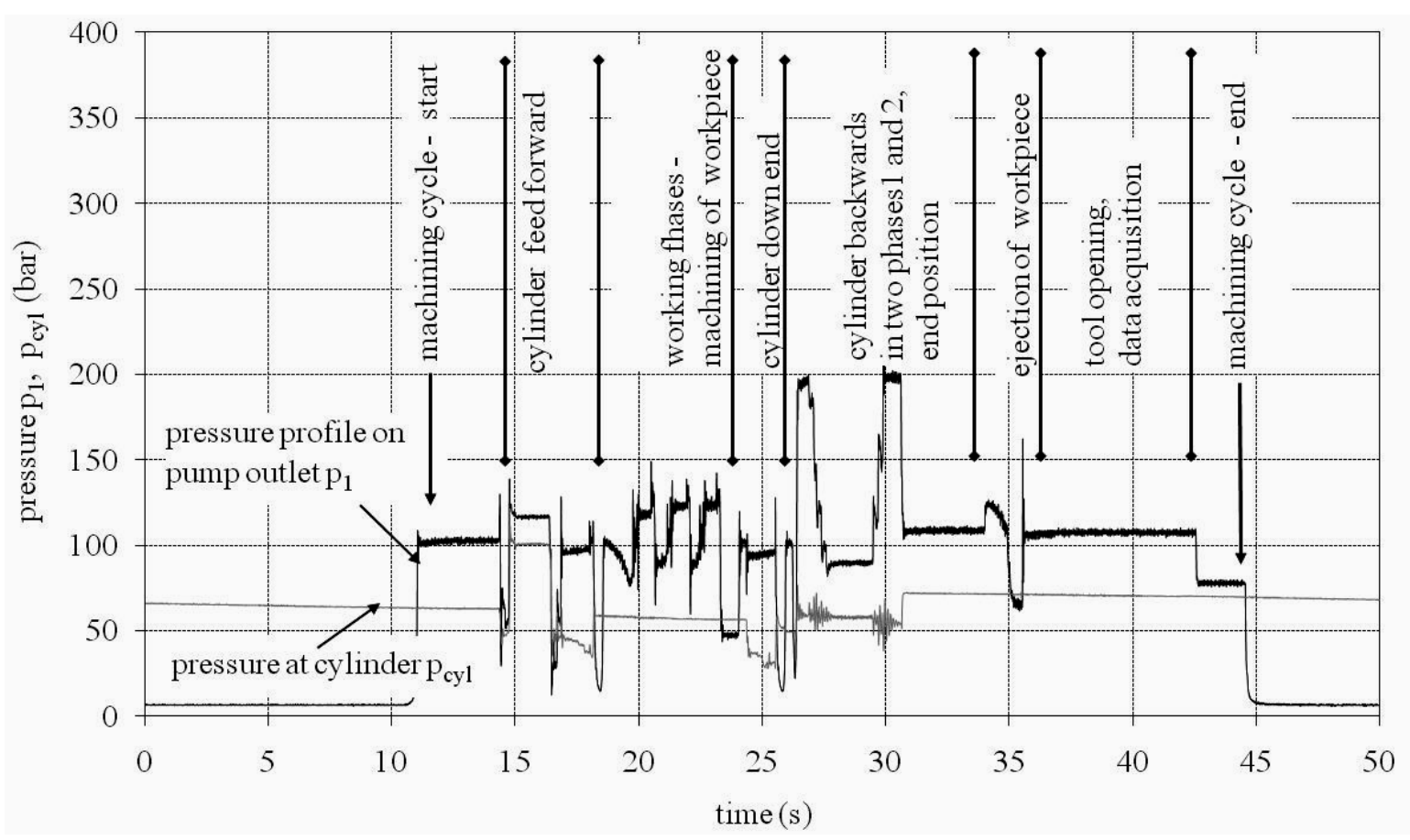

Figure 2: Pressure profile over the working-cycle, with working phases.

For test purposes (verification of the designed mathematical-simulation model), the real pump-pressure profile has to be simplified in the sense that most demanding pressure changes were only reproduced dynamically within the working profile. The simplified profile is presented in Fig. 3.

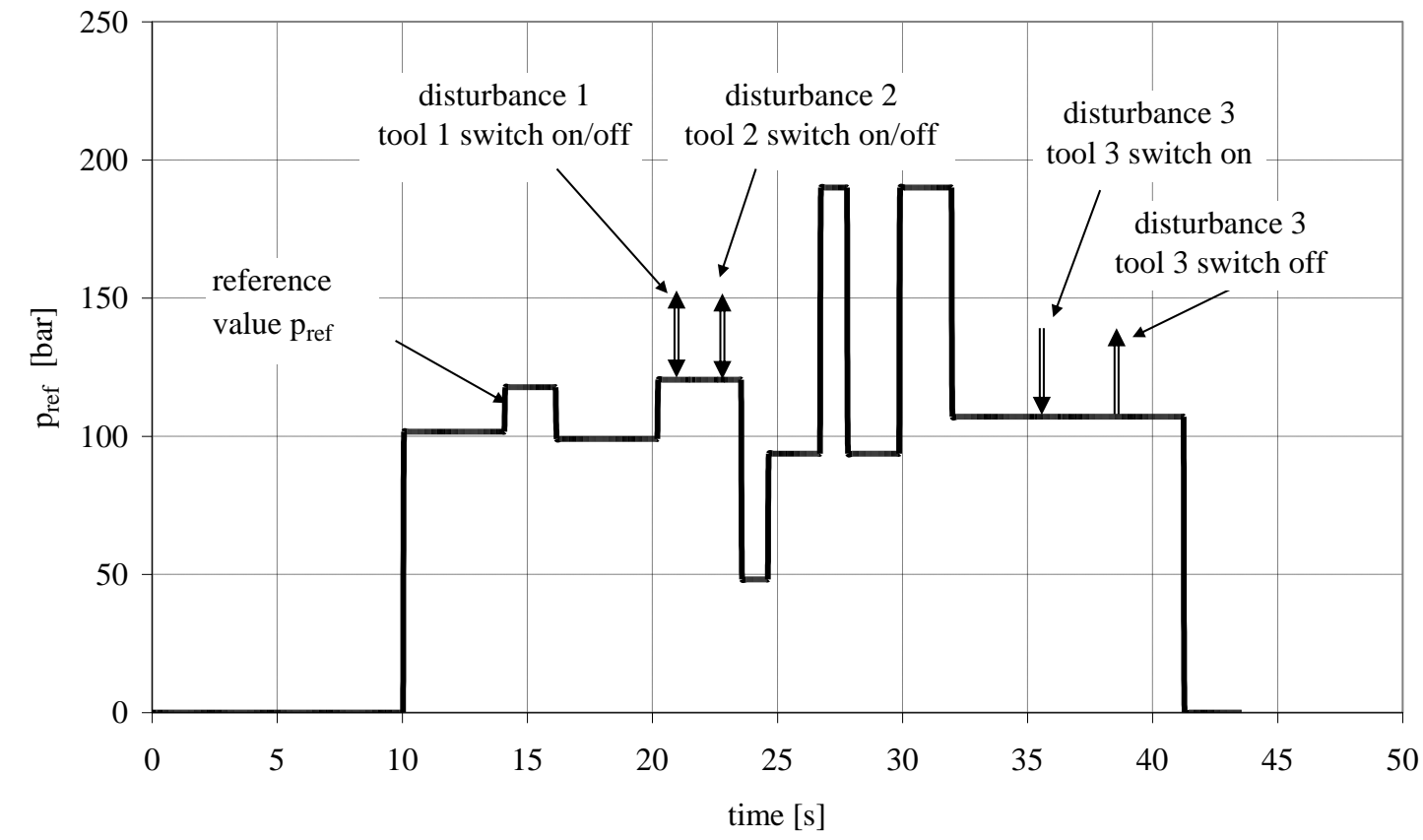

Figure 3: Simplified pressure profile.

All the important time parameters and pressure amplitudes were preserved: start times and end of pressure change, duration of pressure change, pressure amplitude. Only the dynamically unimportant phases were neglected. In addition, a step-change in pressure amplitude was applied representing the dynamics of the most demanding case, for testing the control concept's dynamics. 
A special test-rig, as presented in Fig. 4, was designed for model verification. A selection of those components to be used on the real machine was introduced for the test-rig.

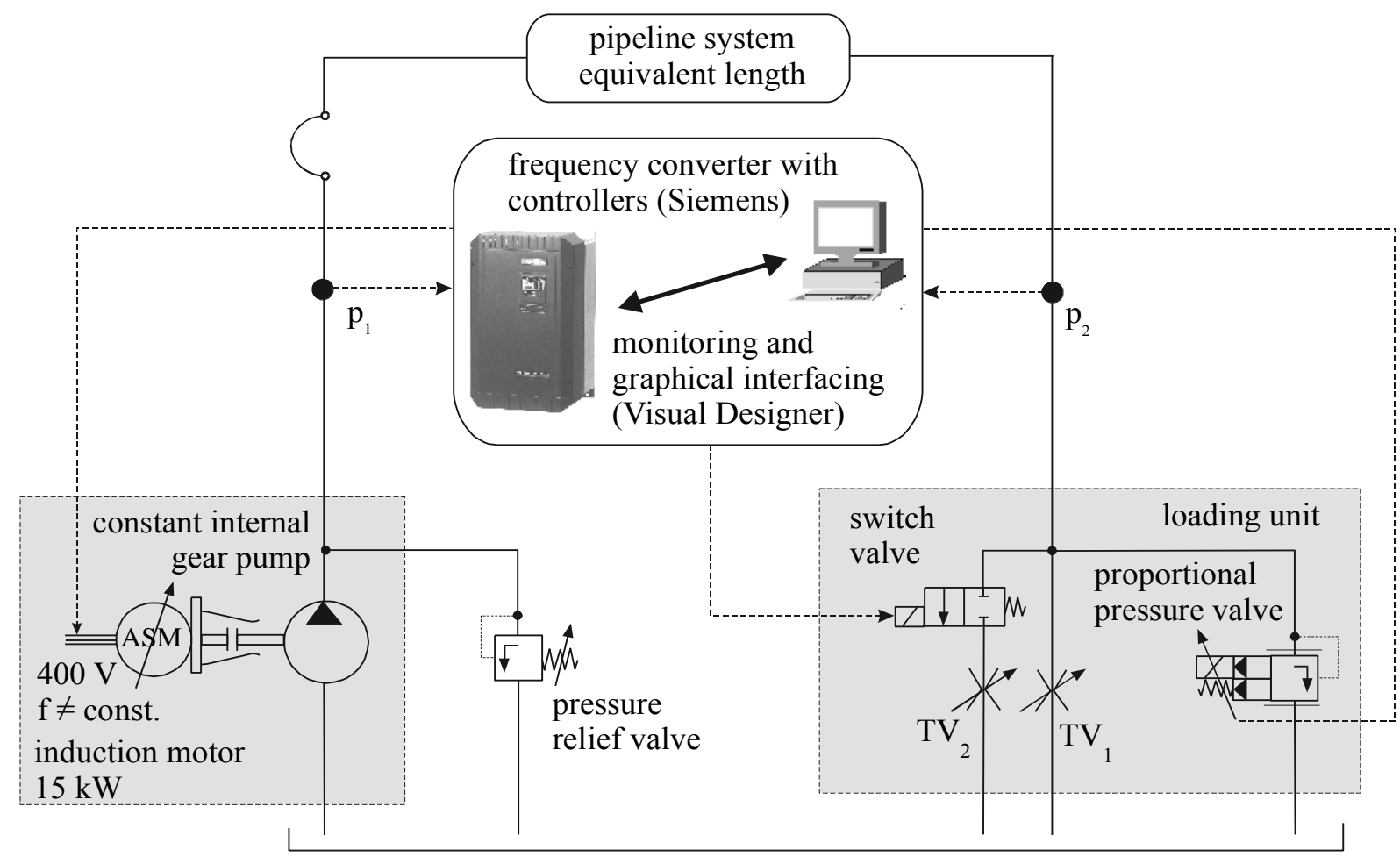

Figure 4: Structure of the equivalent test-rig.

The press-brake hydraulic test system consisted of an internal gear-pump (PGF3 31/032RE07VE4, Bosch-Rexroth) driven by a $15 \mathrm{~kW}$ speed-controlled electric motor. The electric motor was driven by an appropriate frequency converter (Midimaster vector 6SE32, Siemens). The pump supplied a hydraulic pipeline system of equivalent length and dimensions.

Two additional pressure-sensors were applied at the pump-outlet port $\left(\mathrm{p}_{1}\right)$, and at the end of the pipeline system $\left(\mathrm{p}_{2}\right)$, for pressure variation acquisition. All the devices needed for control of the constant pump and variable-speed electric motor (setting of control structure, controllers ...) were integrated into the frequency converter. The settings of the reference values, data acquisition, monitoring, and graphical interfacing, were performed using a personal computer. All the above-mentioned entities must be considered whilst modelling the controlled-system.

\section{MATHEMATICAL MODEL OF SUPPLY SYSTEM}

It is well-known that the modelling of such complex systems, including the interference of hydraulic (hydraulic fluid and mechanics' components), electronics, control techniques and sensor technology, with all their reciprocating influences and interactions (see Fig. 1), is a very demanding task. The subject of different degrees of accuracy when modelling different parts of hydraulic systems has been well described by several authors, where only a simplified modelling is usually applied. These types of mathematical models can produce good results only for a basic simulation and behavioural description of the system. Therefore a detailed description of the complex system and appropriate mathematical model is needed, with all the nonlinearities and specialities of the components. 


\subsection{Speed-controlled electric motor}

Special attention should be paid to an appropriate modelling of the driving concept, especially in the cases of speed-controlled electric motors driven by a frequency converter. The model should adequately consider the asynchronous electric motor and the frequency converter.

Descriptions of dynamical behaviour are mostly used based on a symmetrical description of the induction motor. Very generalized and simplified equations are usually used for easy calculations. Such modelling is presented in Fig. 5.

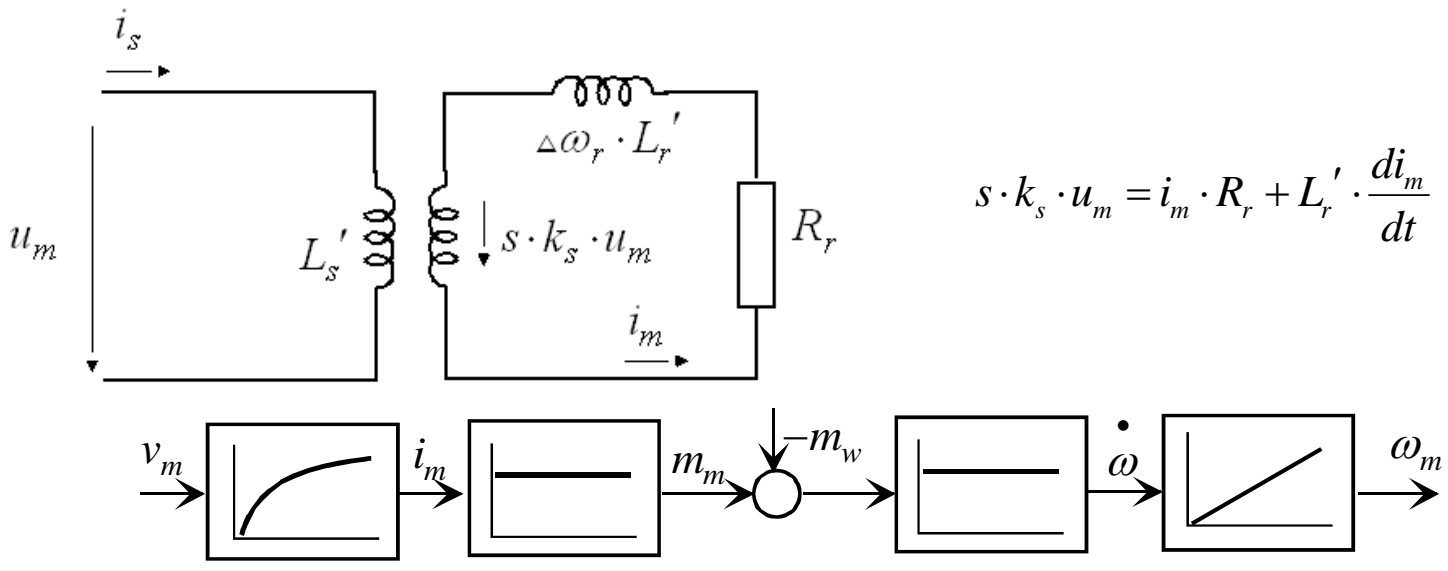

Figure 5: Equivalent circuit of an electric motor with a block diagram for use in simulations.

These types of mathematical models can produce good results for basic simulation and behavioural descriptions of an induction motor, supposing that all the windings of the induction motor are equal. Such a model reduces the number of equations needed to solve the subsequent simulation, but doesn't describe the additional nonlinearity of the power-supply, or the unequal windings. Another approach needs to be used for these kinds of examples.

A better approach is a detailed-description of the winding arrangement for a 3-phase symmetrical induction machine, for the stator and rotor separately. Voltage equations to be used for further mathematical modelling can be obtained using the transformation abc system to dq0 reference framework. In the case of a symmetrical system of rotor and stator windings, these equations can be additionally reduced. A detailed mathematical resp. simulation model of the electric induction machine was designed based on the shown equations.

The winding arrangement for a 3-phase electric motor and the positive directions of the magnetic axes for each winding, are shown in Fig. 6.
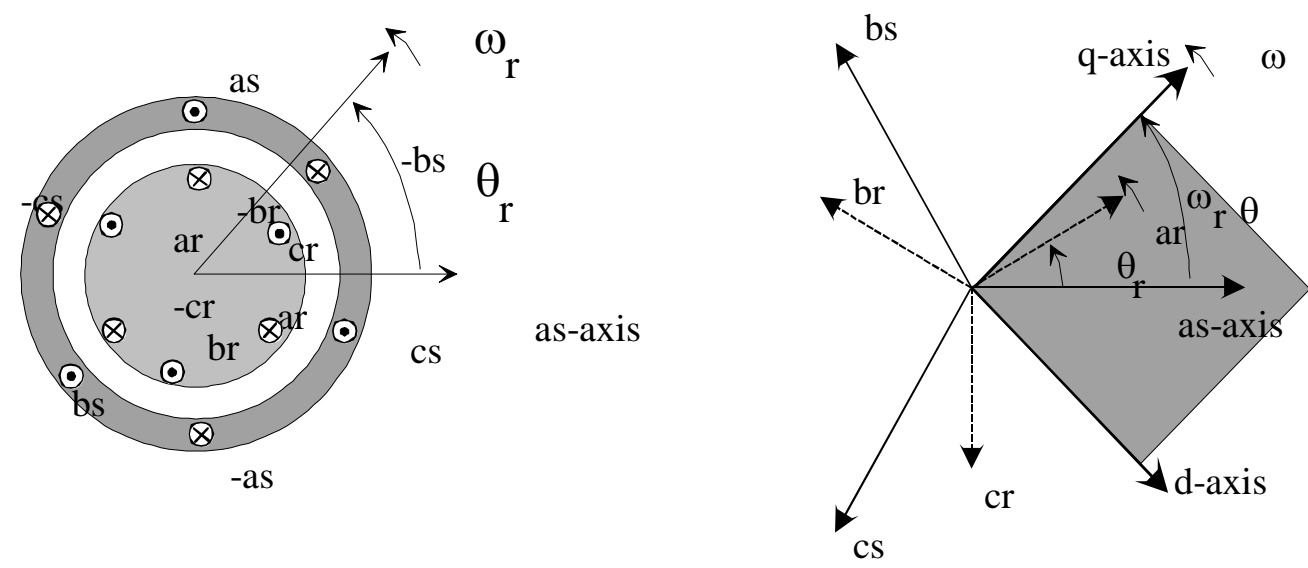

Figure 6: Model of 3-phase symmetrical induction machine. 
The voltage equations for the machine variables may be expressed as:

$$
\mathbf{v}_{a b c s}=\mathbf{r}_{s} \mathbf{i}_{a b c s}+p \lambda_{a b c s} \quad \mathbf{v}_{a b c r}=\mathbf{r}_{r} \mathbf{i}_{a b c r}+p \lambda_{a b c r}
$$

In Eq. (2) the 's' subscript denotes those variables and parameters associated with the stator circuits, and the ' $r$ ' subscript denotes those variables and parameters associated with the rotor circuits. Both $\mathbf{r}_{\mathrm{s}}$ and $\mathbf{r}_{\mathrm{r}}$ are diagonal matrices each with equal non-zero elements, and $\lambda$ flux linkages. For completion, the torque equations are also needed, as shown in Eq. (3). A detailed description of the mathematical models is given in literature [3].

$$
T_{e m}=\frac{3}{2} \frac{P}{2}\left(\lambda_{q r}^{\prime} i_{d r}^{\prime}-\lambda_{d r}^{\prime} i_{q r}^{\prime}\right)=\frac{3}{2} \frac{P}{2}\left(\lambda_{d s}^{\prime} i_{q s}^{\prime}-\lambda_{q s}^{\prime} i_{d s}^{\prime}\right)=\frac{3}{2} \frac{P}{2} L_{m}\left(i_{d r}^{\prime} i_{q s}^{\prime}-i_{q r}^{\prime} i_{d s}^{\prime}\right)
$$

This basic equation can be used within an established equation system when describing the behaviour of electrical values in stator and rotor windings. For complete behaviour, all values are calculated for all three axes q-d-0. All non-linearities can now be observed, as well as different faults (such as an unbalanced operation, Line to-Neutral Fault etc.). Fig. 7 shows the basic model for speed control, with vector PWM invertors.

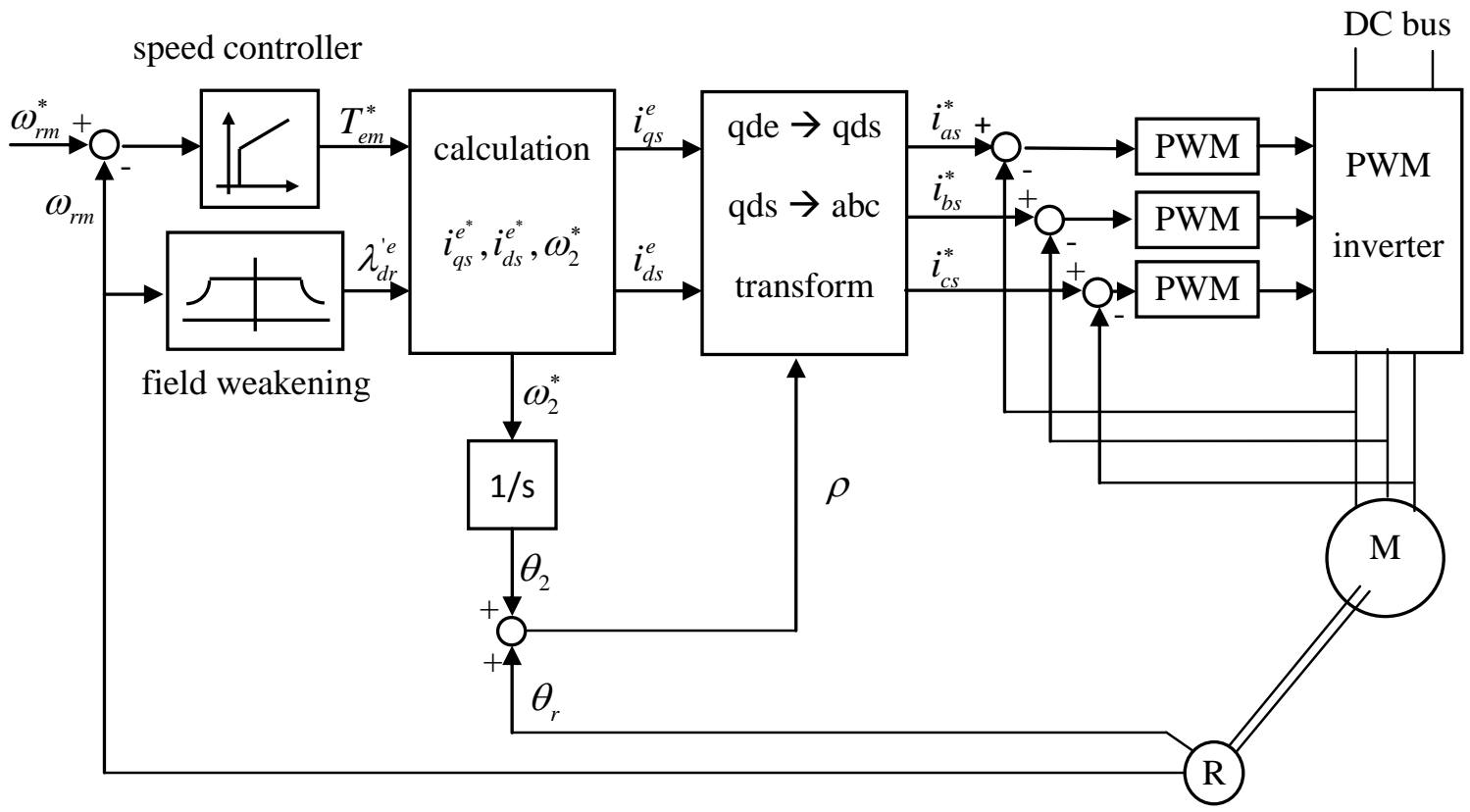

Figure 7: Block scheme of vector speed-control.

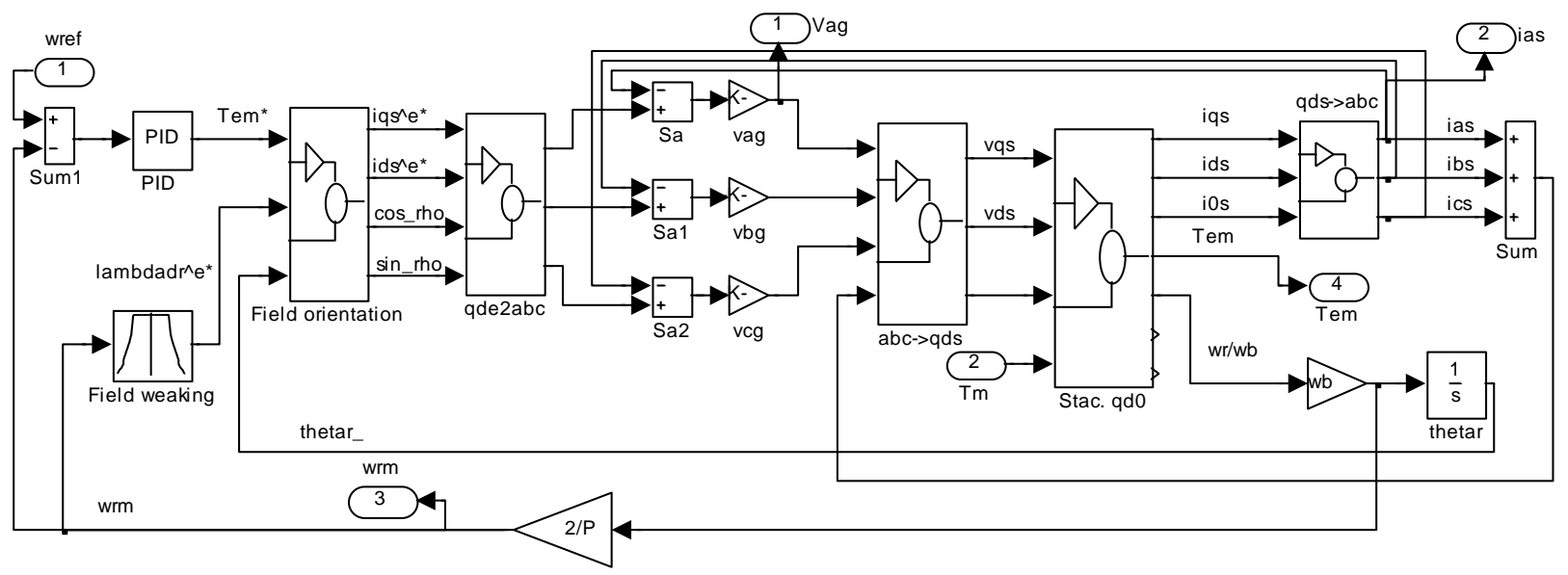

Figure 8: Matlab-Simulink block-scheme for the speed-control of an electric motor. 
A block-scheme was obtained for simulation using Matlab-Simuling, as shown in Fig. 8. The described model can be used for a detailed simulation of a speed-controlled electric motor in combination with the used hydraulic pump as next subsystem, in order to describe the generative part of the entire controlled-supply system.

\subsection{Pump and actuator model}

The next step in this direction was to find an appropriate model of the system's hydraulical components as presented by the modelling of a pump and an actuator.

The modelling of complex-controlled variable pumps, e.g. an electro-hydraulically controlled axial piston pump, is a very demanding task and inevitably leads to a very extensive model. On the other hand, the modelling of constant pumps, as applied in our case, was rather simple. It is acceptable to model the dynamic behaviour of a simple internal gearpump as being proportionally dynamic - Fig. 9 .

The same assumption can be made for an actuator. A hydraulic cylinder as an actuator (or load) controlled by a directional-valve, and presented as a throttle, can be modelled by prescribing its static and dynamic behaviours. The flow behaviour of the throttle is described by Bernoulli's law, where contractions and losses due to the turbulence of the fluid, including any change in the Reynolds number (ratio), need to be considered.

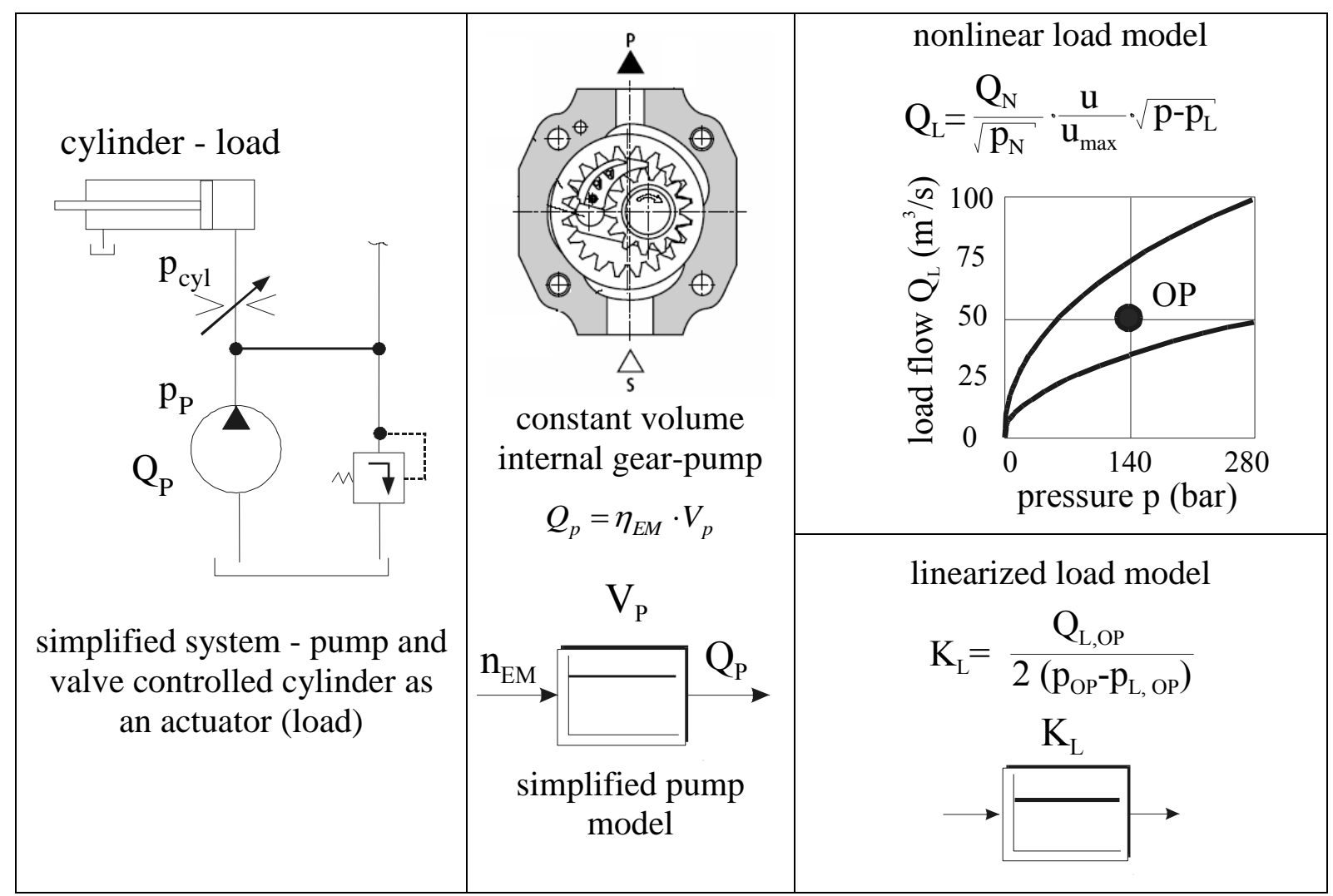

Figure 9: Modelling of pump and the cylinder as a valve controlled actuator.

In general, non-linear models provide accurate modelling of dynamic behaviour but demand long computing times and require a lot of input information, which is often unavailable. When planning and optimising a control-system, where simulation needs to be repeated several times, such models require unacceptably long computing and engineering workloads. Consequently, it is reasonable to apply simplified and linear models of the above subsystems as a proportional dynamic (linearized at the operating point). 


\subsection{Pipeline system}

The pipeline represents the next considered sub-system of the controlled-system. There are many ways of modelling the dynamic behaviour of the fluid within a pipeline: using a socalled continuous model, a discrete model, or with very simplified modelling as a concentrated hydraulic capacity - see Fig. 10.

A continuous model considers the properties of the fluid within the pipeline (that is where the mass, elasticity and friction are continually distributed). A mathematical model is based on the Law of conservation of mass, Navier-Stokes Law, and media properties. Such a model can consist of an unlimited number of degrees of freedom, described by partially differential equations. Solving such a model for simulation and control research purposes is very inconvenient and time-consuming. It requires the applications of tedious integration methods. Therefore a simpler description of pipeline dynamics is frequently used.

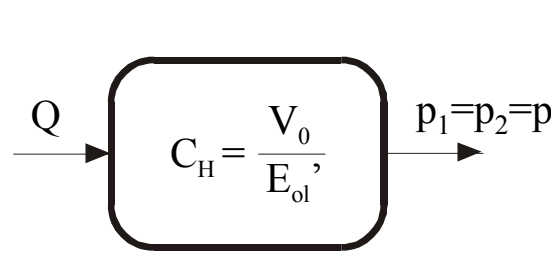

concentrated hydraulically capacity
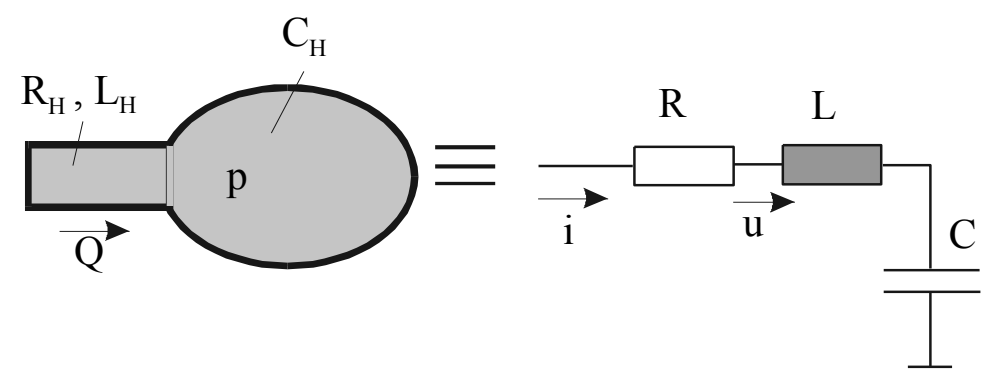

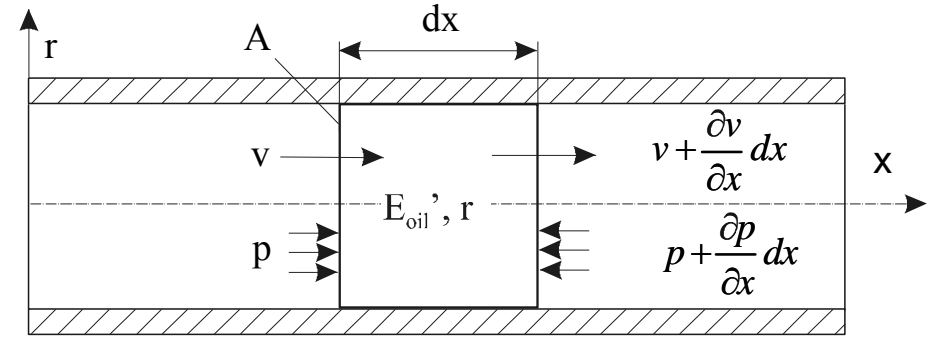

continuum

system with discrete elements: analogue to electro-techniques

Figure 10: Different approaches when pipeline-system modelling.

The simplest way to model the pipeline is to describe it as a concentrated capacity: It has no length, resistance, the fluid within it does not oscillate - the pipeline has no dynamics. In such a model, the pipeline is treated as a homogenous vessel or as fluid closed within a container with no geometric shape (no length and cross-section), no resistance, and no inductivity. No material properties of the fluid are considered. Such a model only provides only approximate results.

In order to improve the model's accuracy, it is reasonable to model pipeline using discrete elements - analogy with electrical engineering - a so-called quadripole equation. The idea of such modelling is based on reducing the number of degrees of freedom. Pressure and volume variation is only monitored at the beginning and at the end of the pipeline. The whole pipeline is modelled by a group of connected sections which oscillate and are elastically connected.

The mathematical model of dynamic behaviour consists of a system of simple differential equations. It can quite easily be applied for simulations or control engineering research. The simulation results from such models are reasonably accurate, and depend on the number of applied discrete elements. 


\subsection{Control-system design}

When constant-pump and speed-controlled electric motors are used for pressure (or for flow as well as power) control, there are two active control-loops: the speed-control loop of the electric motor as a secondary (internal) control-loop, and the pressure-control as a primary control-loop. Such a solution is known as cascade-control.

In the case of cascade-control, two variables are monitored, pressure $p$ and electric motor turning speed $n$. Both values are measured and controlled. A principal block diagram of pressure control in the case of a constant-pump is shown in Fig. 11.

In the case of cascade-control, special attention should be paid to choosing an appropriate type of controller (see e.g. [6], [9]). It is important to ensure that the selected secondary-controller provides sufficient control-loop dynamics, which must be as fast and stable as possible, over the whole expected operational range. It is important to ensure that the selected secondarycontroller provides sufficient control-loop dynamics, which must be as fast and stable as possible, over all the expected operational range. Consequently, a P-controller was used, providing proportional dynamics without delays.

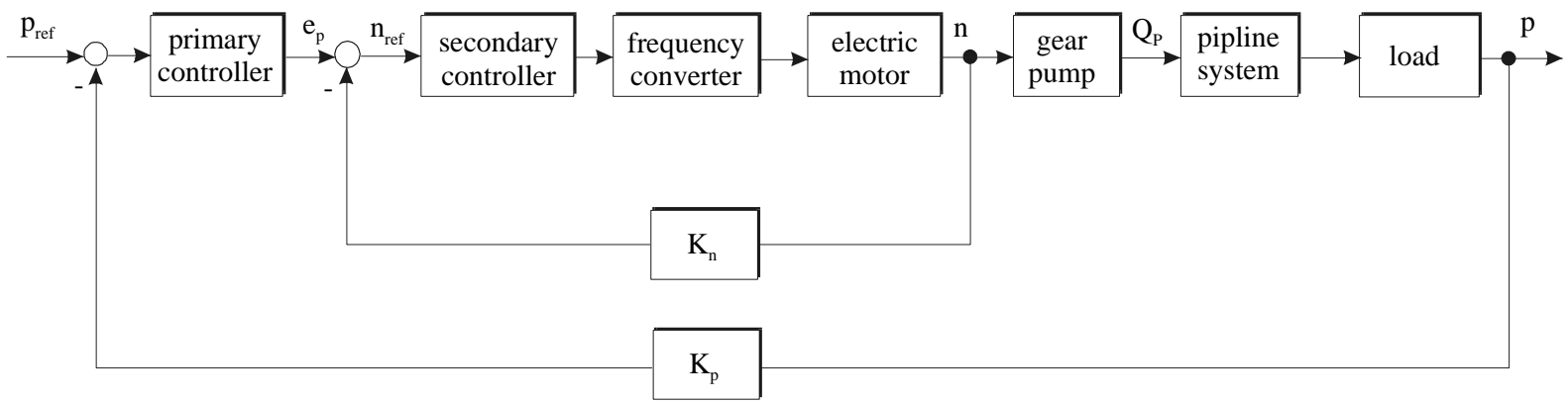

Figure 11: Principal block diagram of discussed cascade-control.

The primary-controller must allow for optimal pressure-control behaviour. In the case under consideration, a P-controller was used in the secondary-loop, and a PID-controller in the primary control-loop. More appropriate dynamic behaviour can be expected when both secondary and primary-controllers are applied,. The application of a secondary controller allows for faster disturbance handling in the secondary-loop.

\section{SIMULATION OF A PRESS BRAKE SUPPLY SYSTEM}

All the represented sub-systems of the discussed controlled press-brake supply system using speed-controlled electric motor in combination with a constant internal gear-pump, must be combined into the simulation model of the entire supply system.

Matlab-Simulink software was used as in the case of modelling the shown electric motor with frequency converter (see Fig. 8). The simulation block scheme of the entire system with all mentioned subsystems: induction motor with frequency converter, a constant pump (as a proportional dynamic), which supplies the pipeline system, is shown in Fig. 12.

In addition to the already-mentioned subsystems, some further subsystems are shown in Fig. 12, for example, a subsystem for describing load changes: a reference value generator (according the simplified pressure profile depicted in Fig. 3), and controllers.

The obtained pressure profiles, as the result of simulation, resulting from the detailed simulation of the entire system using optimised secondary and primary controllers, are presented in Fig. 13. It can be concluded, that the suggested control-concept, using a speed- 
controlled electric motor with an internal gear-pump, enables a sufficient and precise dynamically-achieved pressure profile, promptly following the required reference values.

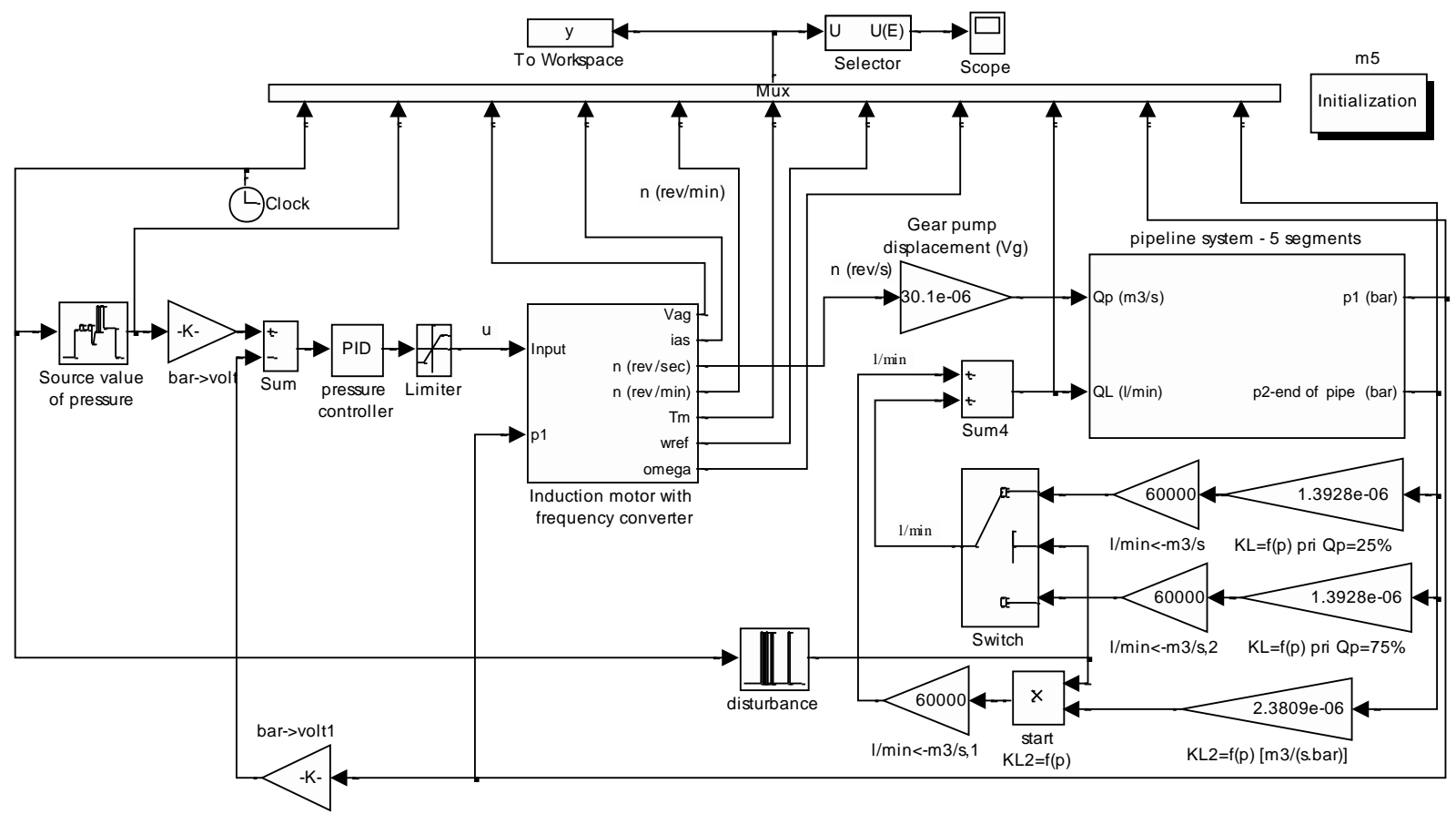

Figure 12: Simulation configuration of the discussed controlled supply-system.

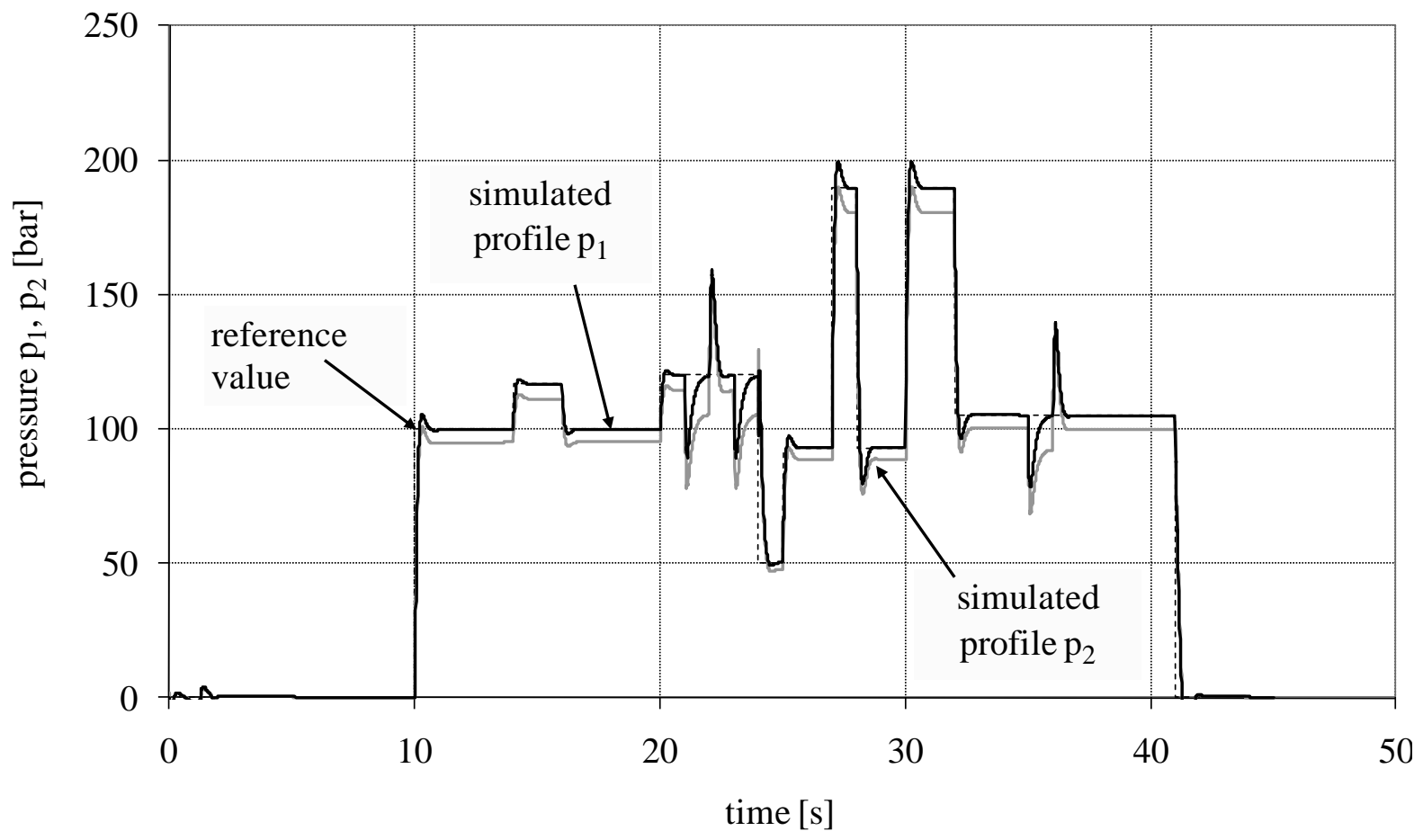

Figure 13: Pressure profiles obtained by simulation.

\section{EXPERIMENTAL VERIFICATION OF SIMULATION RESULTS}

The accuracy of the developed simulation model of the press-brake controlled supply system should be proved by experiment. For this purpose the components used and described in the 
simulation models were used: electric motor, internal gear-pump, original controllers, installed in the existing frequency converter, ... It was necessary to configure the control structure and set appropriate controller parameters, in order to meet the requirements of the described cascade control. Definition and optimisation of both controllers was done, in accordance with the principles of the control technique: always in a stable and optimally steady state, and with dynamical behaviour. The above-mentioned necessary tasks were also done in compliance with the manufacturer's instructions for the applied frequency converter.

As a secondary controller, a PI-controller was used to control speed, whereas the integral parameter was set as low as possible (to achieve behaviour similar to the suggested simple Pcontroller). As primary controller, a PID-controller was used to control the pressure, whereas a higher integral parameter was set to achieve pressure control without steady state error.

A simplified pressure profile (as shown in Fig. 3), was generated, using Visual Designer software. The same software tool was used for all other graphical interfacing with the monitored system.

The actual profile of the achieved control values, using the suggested control concept, is shown in Fig. 14. The variable flow of the constant pump and power is presented in addition to the pump pressure profile. The latter two profiles were calculated from the actual turning speed and geometrical volume of the pump, regarding the influence of the compressibility and temperature of the media.

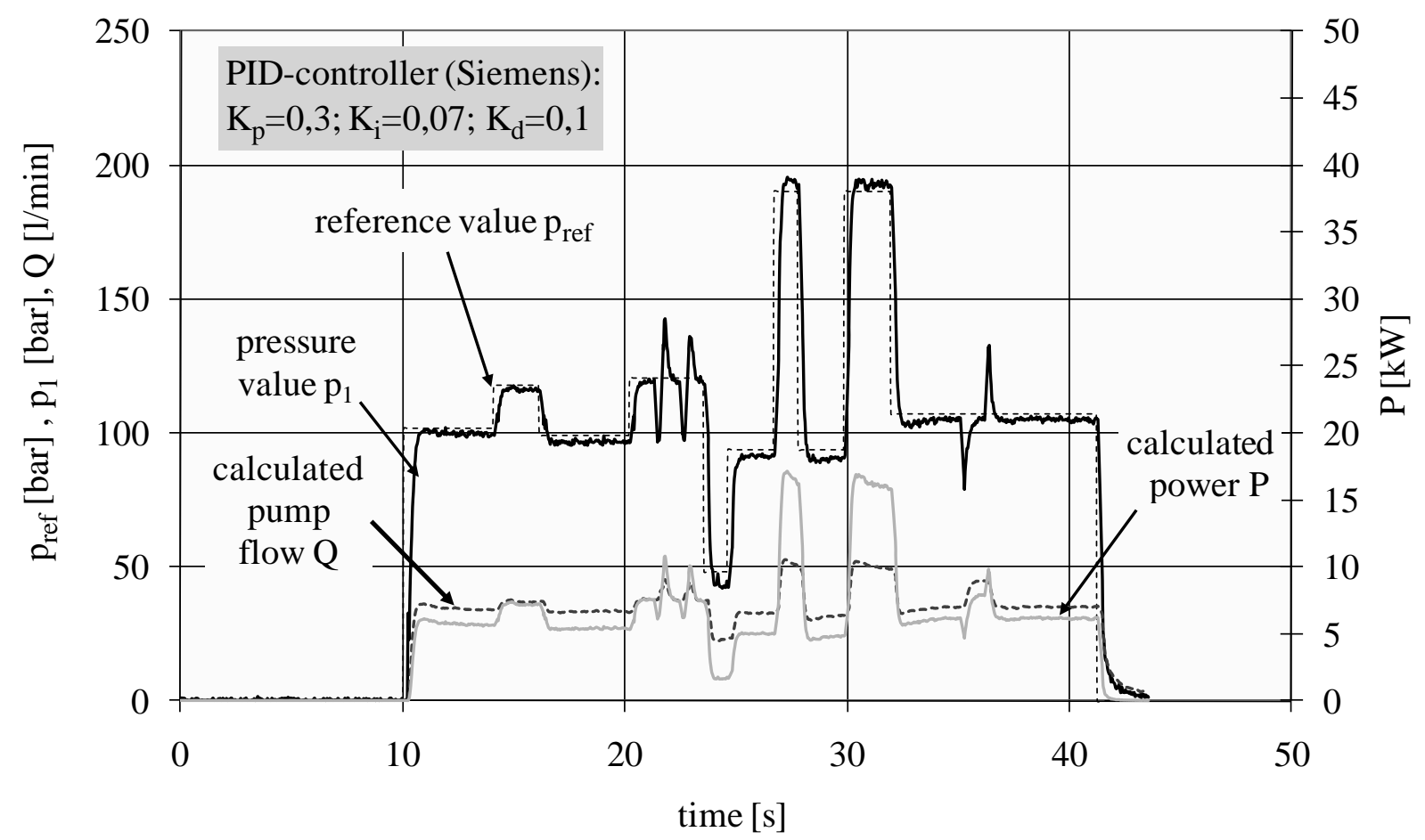

Figure 14: Experiment: pressure, flow and power distribution in the case of a speed controlled motor and internal gear pump.

The experimental results proved that model accuracy of the complex multiphysics model of the drive and the selection of components for the supply systems fulfilled the expectations set for a hydraulic press-brake and encouraged further activities in the sense of supply-system implementation with speed-controlled induction motors, in the field of metal-forming machines. 


\section{CONCLUSION}

The presented work dealt with the modelling and simulation of a modern controlled-hydraulic supply system, where the control of flow or pressure (also control of power) is achieved using a constant pump, in combination with a speed-controlled induction motor. The suggested drive concept was applied in a press-brake.

The discussed system represents an example of a multitechnological mechatronic system, where different technologies met together: electrical, electronic, sensor technology, control technology in combination with hydraulic drive technology with all its specific characteristics. The modelling of such systems leads to a very complex mathematical and simulation model, which must be suitable for engineering use, to assume the use of available software and, of course, provide enough details about what occurs in any part of the system and with the system as a whole.

The results, obtained from a computational model of the entire system showed that the suggested selection of drive components was appropriate for a new drive-design. The experimental results confirmed the findings obtained by the developed mathematical model. So the presented theoretical and experimental study can serve as a fundamental analysis for the implementation of supply systems with speed-controlled induction motors, in the field of metal forming and similar machines equipped with hydraulic supply system.

\section{REFERENCES}

[1] Rühlicke, I. (1997). Elektrohydraulische Antriebssysteme mit drehzahlveränderbarer Pumpe (Eng.: Elctrohydraulic drive systems with speed controlled Pumps), Dissertation, TU Dresden

[2] Neubert, T. (2002). Untersuchungen von drehzahlveraenderbaren Pumpen (Eng.: Research of speed controlled pumps), Dissertation, TU Dresden

[3] Kastrevc, M. (2003). Analysis of dynamic of drive concept using induction electromotor and hydraulic gear pump, Dissertation, Univ. of Maribor

[4] Helbig, A. (2002). Injection Molding Machine with Electric-Hydrostatic Drives, $3^{\text {rd }}$ International Fluid Power Colloquium Aachen, Vol. I, 67-81

[5] Helduser, S. (2003). Improved Energy Efficiency in Plastic Injection Molding Machines, $8^{\text {th }}$ Scandinavian International Conference on Fluid Power, SICFP'03, Tampere, Finland

[6] Lovrec, D.; Ulaga, S. (2007). Pressure control in hydraulic systems with variable or constant pumps?, Experimental Techniques, Vol. 31, No. 2, 33-41, doi:10.1111/j.1747-1567.2007.00146.x

[7] Bonefeld, R.; Lebert, C. (2008). Adaptive pressure control using variable speed pump; $6^{\text {th }}$ International Fluid Power Conference, Dresden, Workshop Proceedings, 151-164

[8] Ristic, M. (2008). Conversant technology - New key aspects: Development of variable speed drives, $6^{\text {th }}$ International Fluid Power Conference, Dresden, Proceedings, 93-108

[9] Föllinger, O. (1994). Control Technique, Hüthing, Heidelberg 